\title{
A Bounded Uncertainty Approach to Cooperative Localization Using Relative Bearing Constraints
}

\author{
Camillo J. Taylor and John Spletzer
}

\begin{abstract}
This paper describes an approach to cooperative localization which finds its roots in robust estimation, employing an unknown-but-bounded error model for sensor measurements. In this framework, range and bearing measurements obtained by the robots are viewed as constraints which implicitly define a set of feasible solutions in the joint configuration space of the robot team. The scheme produces bounded uncertainty estimates for the relative configuration of the team by using convex optimization techniques to approximate the projection of this feasible set onto various subspaces of the configuration space. The scheme can also be used to localize distributed sensor nodes.

An important advantage of the proposed approach is that it is able to produce bounded uncertainty estimates for the relative configuration of the robots even in the case where the relative orientations of the robots are completely unknown. This is an important practical advance since errors in relative orientation are often a major contributor to positioning uncertainty in multi-robot localization schemes.
\end{abstract}

\section{INTRODUCTION}

Localization is a critical base level capability for mobile robots and sensor networks enabling numerous other technologies including mapping, manipulation, and target tracking. It is not surprising then that considerable research effort has been directed at this problem [1], [2], [3], [4], [5]. Within this realm of research, there is a narrower yet still significant focus on cooperative localization for multi-robot teams [6], [7], [8]. In this paradigm, groups of robots combine sensor measurements to improve localization performance. This approach is motivated by the fact that robots within a team can often identify one another and communicate sensor measurements, such as relative range and bearing readings.

In this paper, we revisit our bounded uncertainty approach to the multi-robot localization problem initially proposed in [9]. Conceptually, the idea is that sensor measurements induce constraints on the configuration space of the robot team. Merging these constraints induces a feasible set on the configuration space that represents the set of formation poses that are consistent with all of the available sensor measurements. Estimates for the uncertainty in various parameters of

C.J. Taylor is with the Department of Computer and Information Science, University of Pennsylvania, 3330 Walnut St, Philadelphia PA. 19104, USA cjtaylorecis. upenn. edu

J. Spletzer is with the Department of Computer Science and Engineering, Lehigh University, 19 Memorial Drive West, Bethlehem PA. 18015,USA spletzerecse. lehigh.edu

This material is based upon work supported by the National Science Foundation under Grant Nos. 9875867 and 0130858 . Any opinions, findings, and conclusions or recommendations expressed in this material are those of the author(s) and do not necessarily reflect the views of the National Science Foundation. the team's configuration such as the absolute position of a single robot, or the relative positions of two or more nodes can then be obtained by projecting this feasible set onto appropriately chosen subspaces of the configuration space. Unfortunately, recovering these projections exactly is quite cumbersome - requiring exponential time in the number of constraints. Instead, we propose a scheme to approximate these projections using modern convex optimization techniques.

A significant shortcoming of our previous work was the requirement that each of the robots have a sensor which provided an orientation estimate with respect to a common reference frame (e.g., a compass or solar sensor). Such an assumption makes the math more tractable, and is often employed in multi-robot localization schemes [10], [11], [12], [8]. The primary contribution of this work is a means for localization using bearings sensors (e.g. cameras) that can recover the relative robot positions without such an orientation sensor. The approach can also accommodate range measurements, and has a computational complexity scaling polynomially in the number of robots. Furthermore, the workload is readily distributed requiring only the communication of sensor measurements between nodes.

The remainder of this paper is organized as follows: Section II provides a literature review. A discussion of the localization approach follows in Section III. Experimental results are presented in Section IV. Finally, a discussion of the approach's merits along with directions for future work are outlined in Section V.

\section{RELATED WORK}

In our previous work [9], we described bounded uncertainty approaches that could be used in the case where the robots could measure their orientation with respect to a common frame of reference. In this case, the available range and bearing measurements can be converted into linear inequality constraints on the joint configuration space of the robots. In this work we extend the same basic approach to deal with feasible sets that are defined in terms of quadratic constraints. This advance allows us to deal with upper and lower bound range constraints and the relative bearing constraints which will be described in the sequel. This in turn allows us to handle cases where the relative orientations of the robots are completely unknown.

This work relates to cooperative localization techniques, where sensor measurements from multiple robots are integrated to estimate uncertainties in absolute or relative position. The first forays in this area employed direct localization 
techniques, where the relative robot poses could be solved analytically [13], [14]. These efforts were followed by other researchers relying upon Bayesian techniques [6], [7], [12], [8] for pose estimation. A primary advantage of Bayesian approaches is that they not only propagate a state estimate over time, but also the uncertainty associated with the state estimate.

Our proposed approach also provides an estimate of the uncertainty in the robot position. However, this is represented instead in terms of feasible sets in the configuration space and measurements are combined via set intersection. An important advantage of this approach is that we do not need to account for dependencies or correlations between measurements to combine them using set intersection. We can contrast this with the bookkeeping required to properly account for dependencies between estimates in a Bayesian framework. This is a particularly important advantage in the context of cooperative localization where the measurements of relative range and bearing induce a complex web of dependencies among the node position estimates. Another advantage of the approach is that it avoids the linearizations inherent in approaches based on the Kalman filter. These linearizations can be particularly problematic when they are used to model the effects of uncertainties in the robots' orientations. In the sequel we will describe a bounded uncertainty estimation scheme that can produce accurate estimates for the robots configuration without any prior estimates for the robots relative orientation. However, these benefits are not without cost as the computational complexity exceeds that of Kalman Filter approaches in both theory and practice.

The proposed approach shares the same general philosophy as the set based SLAM algorithm proposed by Di Marco, Garulli, Giannitrapani, and Antonio Vicino [21]. In our work the feasible set is represented implicitly in terms of a set of inequality constraints which is a significant point of departure. It also explicitly deals with situations where the robot orientations are unknown through the use of higher order constraint equations on the feasible set.

Cooperative robot localization is also strongly related to the problem of localizing wireless sensor nodes. This has received significant attention in the sensor networks literature and a number of effective approaches have been proposed [15], [16], [17], [18], [19]. Perhaps most related is the work of Biswas, Aghajan and Ye [18] who have also used relative bearing measurements to help estimate the configuration of sensor nodes [20]. Our work extends these approaches by showing how relative bearing constraints can be used to produce useful guaranteed bounds on the relative positions of the node. Importantly, this allows us to tackle localization problems that were previously beyond the reach of bounded uncertainty techniques.

\section{THE LOCALIZATION APPROACH}

The basic elements of the localization framework are diagrammed in Figure 1. Here a set of robots or sensor nodes equipped with range and/or bearing sensors are located on the plane. The edges in the figure indicate available

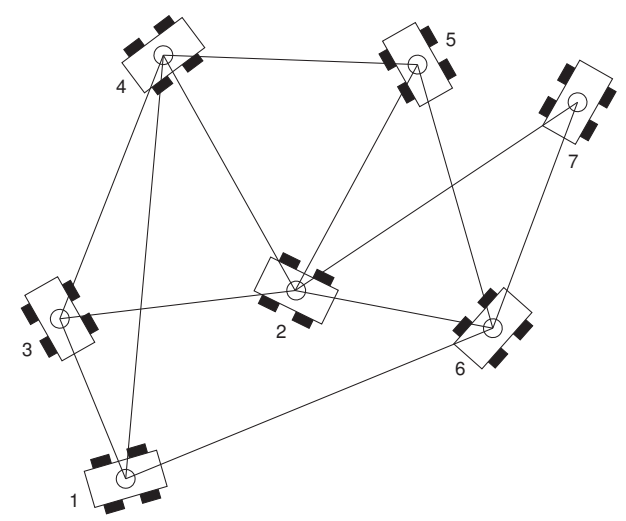

Fig. 1. A set of robot or sensor nodes located in a 2D workspace. The edges between the nodes indicate available sensor measurements, that is an edge between nodes $\mathrm{i}$ and $\mathrm{j}$ indicates that robot $\mathrm{i}$ can measure the range and or bearing to node $\mathrm{j}$ from its current position.

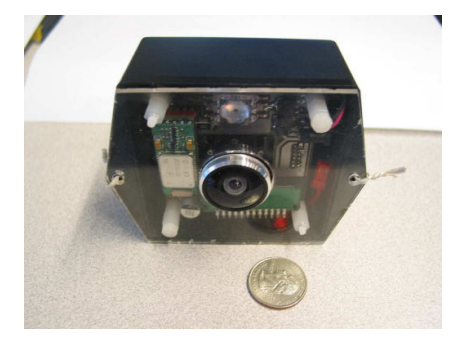

Fig. 2. This smart camera node, which is equipped with an accelerometer, can measure the relative bearing to other sensor nodes or robots.

relative measurements, that is an edge between nodes $i$ and $j$ indicates that robot $i$ can measure the range and or bearing to node $j$ from its current position. Note that the edges in this graph will, in general, be directed.

For example, the proposed scheme can be employed to localize ensembles of smart camera sensor nodes such as the one shown in Figure 2. These nodes are capable of measuring the relative bearing to other sensor nodes in the vicinity using image measurements and their orientation with respect to gravity using an onboard accelerometer [19]. Our method can then be used to gauge the relative positions of the nodes in the horizontal plane based on this information.

Let $\mathcal{C} \subset \mathbb{R}^{2 n}$ denote the configuration space of our robot team. Let $\mathbf{x}=\left(\begin{array}{lllllll}x_{1} & y_{1} & x_{2} & y_{2} & \cdots & x_{n} & y_{n}\end{array}\right) \in \mathcal{C}$ denote the current configuration of the ensemble. Note that $\mathbf{x}$ is simply the concatenation of the coordinates of the $n$ robots. Note also that we are purposely vague about the frame of reference to which these coordinates are to be measured. Depending on what parameters we are trying to estimate, various choices will be more or less convenient. Hence we defer this decision until the parameter estimation phase.

\section{A. Generating Sensor Constraints}

In this framework, we assume that we can bound the error in the range and bearing measurements. Each such measurement is then viewed as a constraint on the possible values of the configuration vector $\mathbf{x}$. This section focuses on 


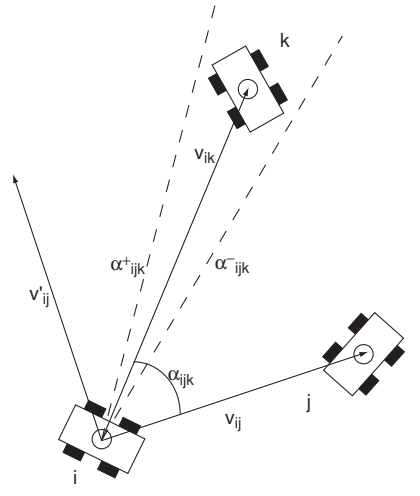

Fig. 3. Robot $i$ simultaneously observes two other robots, $j$ and $k$. The measurement for the angle $\alpha_{i j k}$ subtended at $i$ can be used to constrain the configuration of the team.

the transformations required to realize this.

In the sequel we will let the vector $v_{i j} \in \mathbb{R}^{2}$ denote the displacement between nodes $i$ and $j$. This vector can be expressed as a linear function of the configuration vector $\mathbf{x}$ as follows: $v_{i j}=\left(\boldsymbol{D}_{j}-\boldsymbol{D}_{\boldsymbol{i}}\right) \mathbf{x}$, where $\boldsymbol{D}_{\boldsymbol{m}} \in \mathbb{R}^{2 \times 2 n}$ denotes the sparse projection matrix that extracts the coordinates of node $m$ from the configuration vector $\mathbf{x}$ in $\mathbb{R}^{2 n}$.

Importantly, all of the constraints that we will describe in the following subsections can be expressed as quadratic inequalities of the configuration vector $\mathrm{x}$, that is they can all be written in the form $\mathbf{x}^{T} A \mathbf{x}+b^{T} \mathbf{x}+c \leq 0$ for some $A \in \mathbb{R}^{2 n \times 2 n}, b \in \mathbb{R}^{2 n}, c \in \mathbb{R}$. A constraint of this form is termed convex if the matrix $A$ is positive semidefinite or 0 since inequalities of this form define convex regions in the configuration space. Note that the set of quadratic inequalities subsumes the set of all linear inequalities.

1) Relative Bearing Constraints: In Figure 3 Robot $i$ simultaneously observes two other nodes, $j$ and $k$. The vectors $v_{i j}$ and $v_{i k}$ denote the displacements between nodes $i$ and $j$ and $i$ and $k$ respectively. Multiplying $v_{i j}$ by the constant rotation matrix $R_{90}=\left(\begin{array}{cc}0 & -1 \\ 1 & 0\end{array}\right)$ yields a second vector $v_{i j}^{\prime}$ with the same magnitude as $v_{i j}$ but rotated by 90 degrees counter clockwise. If we take inner products between the vector $v_{i k}$ and the vectors $v_{i j}$ and $v_{i j}^{\prime}$ we can form another vector which encodes the angular separation, $\alpha_{i j k}$, between $v_{i j}$ and $v_{i k}$ :

$$
\begin{aligned}
\left(\begin{array}{c}
v_{i k} \cdot v_{i j} \\
v_{i k} \cdot v_{i j}^{\prime}
\end{array}\right) & =\left(\begin{array}{c}
v_{i k}^{T} v_{i j} \\
v_{i k}^{T} R_{90} v_{i j}
\end{array}\right) \\
& =\left\|v_{i k}\right\|\left\|v_{i j}\right\|\left(\begin{array}{c}
\cos \alpha_{i j k} \\
\sin \alpha_{i j k}
\end{array}\right)
\end{aligned}
$$

The available bearing measurements would constrain this vector to the sector defined by the two angles $\alpha_{i j k}^{-}$and $\alpha_{i j k}^{+}$ These constraints are expressed in the following inequalities.

$$
\begin{aligned}
& \left(\sin \alpha_{i j k}^{-}-\cos \alpha_{i j k}^{-}\right)\left(\begin{array}{l}
v_{i k} \cdot v_{i j} \\
v_{i k} \cdot v_{i j}^{\prime}
\end{array}\right) \leq 0 \\
& \left(\sin \alpha_{i j k}^{+}-\cos \alpha_{i j k}^{+}\right)\left(\begin{array}{l}
v_{i k} \cdot v_{i j} \\
v_{i k} \cdot v_{i j}^{\prime}
\end{array}\right) \geq 0
\end{aligned}
$$

Using Equation 1, both of these constraint equations can be rewritten as quadratic functions of the parameter vector $\mathrm{x}$.

$$
\begin{aligned}
& v_{i k}^{T}\left(\sin \alpha_{i j k}^{-} I-\cos \alpha_{i j k}^{-} R_{90}\right) v_{i j} \leq 0 \\
& v_{i k}^{T}\left(\sin \alpha_{i j k}^{+} I-\cos \alpha_{i j k}^{+} R_{90}\right) v_{i j} \geq 0
\end{aligned}
$$

Note that these constraints will, typically, not be convex functions of $\mathrm{x}$. Note also that these constraints do not require any knowledge of the robots orientation with respect to an absolute frame of reference. This is an important advance since it allows us to directly exploit the kinds of relative bearing measurements that can be derived easily and accurately from imaging sensors without requiring a compass or any other orientation estimation scheme.

Relative bearing measurements have also been employed in a different manner for bounded uncertainty single robot localization problems by Briechle and Hanebeck [22]. Biswas, Aghajan and Ye also make use of relative bearing constraints in their work but use a different mathematical formulation based on the circle defined by the three points. In contrast our formulation provides equations which directly bound the relative configuration of the nodes.

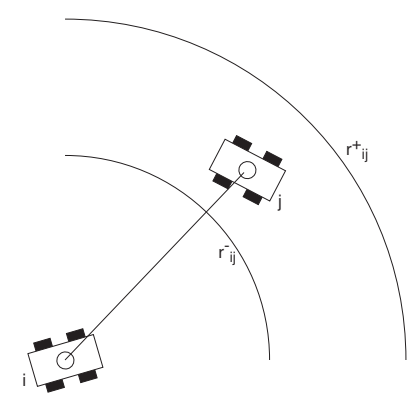

Fig. 4. Range measurements with bounded error constrain the distance between two nodes $i$ and $j$.

2) Range Constraints: Figure 4 depicts the annulus of feasible configurations induced by constraints on the range between nodes $i$ and $j$. The constraints induced by a bounded error range measurement can be expressed quite simply [15] in terms of the configuration vector $\mathbf{x}$. Here we again note that the vector displacement between nodes $i$ and $j$, $v_{i j}$ can be expressed as a linear function of the global configuration vector x. i.e. $v_{i j}=\left(\boldsymbol{D}_{j}-\boldsymbol{D}_{\boldsymbol{i}}\right) \mathbf{x}$. If $r_{i j}^{+}$and $r_{i j}^{-}$respectively denote the upper and lower bounds on the range measurement, we can derive two quadratic constraints as follows:

$$
\begin{aligned}
v_{i j}^{T} v_{i j} & \leq\left(r_{i j}^{+}\right)^{2} \\
\Rightarrow \mathbf{x}^{T}\left(D_{j}-D_{i}\right)^{T}\left(D_{j}-D_{i}\right) \mathbf{x} & \leq\left(r_{i j}^{+}\right)^{2} \\
v_{i j}^{T} v_{i j} & \geq\left(r_{i j}^{-}\right)^{2} \\
\Rightarrow \mathbf{x}^{T}\left(D_{j}-D_{i}\right)^{T}\left(D_{j}-D_{i}\right) \mathbf{x} & \geq\left(r_{i j}^{-}\right)^{2}
\end{aligned}
$$

Note that the upper bound constraint shown in Equation 6 is a convex function of $\mathrm{x}$ while the lower bound constraint in Equation 7 is concave. 


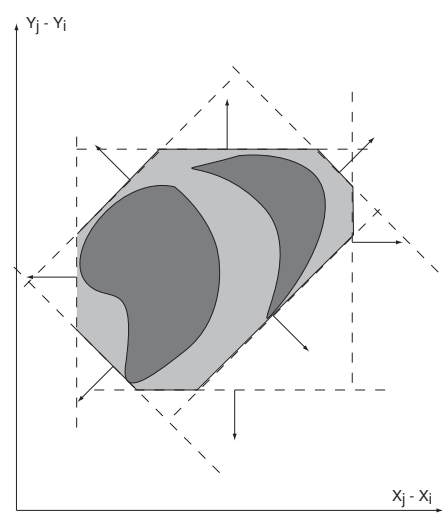

Fig. 5. A convex approximation for the feasible set of displacements (the dark region) can be obtained by bounding the its extent along various search directions. The intersection of the resulting half planes defines a convex polytope (the light gray area) which, by construction, encloses the feasible set.

\section{B. Gauging Team Configurations}

Once a set of constraints has been derived from the available measurements, we can proceed to consider the problem of gauging the positions of the robots with respect to each other. Consider for example the problem of estimating the relative position of node $j$ with respect to node $i$ represented by the displacement vector $v_{i j}$. As discussed earlier, this vector can be expressed as a linear function of the configuration vector $\mathrm{x}$ as follows: $v_{i j}=\left(\boldsymbol{D}_{j}-\boldsymbol{D}_{\boldsymbol{i}}\right) \mathbf{x}$. Hence, we can view the problem of bounding this vector as one of gauging the projection of the set of feasible configurations onto the 2D subspace corresponding to the displacement $v_{i j}$.

We can bound the uncertainty in this displacement vector by choosing search directions parallel to the subspace of interest and bounding the extent of the feasible region in those directions. This amounts to finding bounds for objective functions of the form shown in Equation 8 given the constraints derived from the measurements.

$$
\left.\begin{array}{c}
\qquad\left(\begin{array}{ll}
\cos \theta & \sin \theta
\end{array}\right) v_{i j}=s^{T} \mathbf{x} \\
\text { where } s^{T}=\left(\begin{array}{ll}
\cos \theta & \sin \theta
\end{array}\right)\left(\boldsymbol{D}_{\boldsymbol{j}}-\boldsymbol{D}_{\boldsymbol{i}}\right.
\end{array}\right)
$$

In this case the vector $s \in \operatorname{span}\left(\left(\boldsymbol{D}_{\boldsymbol{j}}-\boldsymbol{D}_{\boldsymbol{i}}\right)^{T}\right)$ corresponds to the search direction in the configuration space.

Figure 5 depicts the most general situation where the feasible set and its projection need not be convex or even connected. In any case, the goal of the localization scheme is to produce a convex polyhedral approximation which bounds the projection of all feasible configurations and, hence, bounds the displacement vector. We see then that the process of bounding the displacement vector relating two nodes, $v_{i j}$, can be reformulated as a sequence of constrained optimization problems which serve to gauge the extent of the feasible region along various search directions. Here we recognize that these optimization problems are actually quadratically constrained quadratic programs (QCQP) since both the objective function described in Equation 8 and the constraint functions described in the previous sections can be written as quadratic functions of the parameter vector $\mathbf{x}$. More precisely, our goal is to solve constrained optimization problems of the following form.

$$
\begin{aligned}
\operatorname{minimize} & \mathbf{x}^{T} A_{0} \mathbf{x}+b_{0}^{T} \mathbf{x}+c_{0} \\
\text { subject to } & \mathbf{x}^{T} A_{i} \mathbf{x}+b_{i}^{T} \mathbf{x}+c_{i} \leq 0 i=1, \ldots, m .
\end{aligned}
$$

Note that although the objective function described in equation 8 is actually a linear function of $\mathbf{x}$, we are in fact able to bound arbitrary quadratic functions of $\mathrm{x}$. This means, for example that we can use precisely the same machinery to bound the square of the distance between nodes $i$ and $j$ which can, clearly, be expressed as a quadratic function of $\mathbf{x}$ as shown in Equation 10 .

$$
\begin{aligned}
\left\|v_{i j}\right\|^{2} & =\left\|\left(D_{j}-D_{i}\right) \mathbf{x}\right\|^{2} \\
& =\mathbf{x}^{T}\left(D_{j}-D_{i}\right)^{T}\left(D_{j}-D_{i}\right) \mathbf{x}
\end{aligned}
$$

Most of the literature on solving QCQPs centers on the special case where the constraint functions are convex which is not, in general, the case here. Nonetheless we can make progress by observing that there are convex relaxations of the original optimization problem that provide useful bounds on the feasible solutions. In this work we use the Lagrangian relaxations described in [23], [24], [25]. The Lagrangian, $L(\mathbf{x}, \lambda)$, and the Lagrangian dual, $g(\lambda)$, of our original optimization problem can be expressed as follows:

$$
\begin{aligned}
L(\mathbf{x}, \lambda)= & \left(\mathbf{x}^{T} A_{0} \mathbf{x}+b_{0}^{T} \mathbf{x}+c_{0}\right)+ \\
& \sum_{i=1}^{m} \lambda_{i}\left(\mathbf{x}^{T} A_{i} \mathbf{x}+b_{i}^{T} \mathbf{x}+c_{i}\right) \\
g(\lambda)= & \inf _{\mathbf{x}} L(\mathbf{x}, \lambda)
\end{aligned}
$$

We can bound the minima of our original optimization problem by finding the maximum of the Lagrangian dual function $g(\lambda)$ over all non-negative values of $\lambda$. Note that the Lagrangian dual will be convex even if the original optimization problem is not. Using Shur complements this optimization problem can be recast as a semidefinite program as follows: maximize $\gamma$ subject to the constraint that the following matrix remains positive semi-definite.

$$
\left[\begin{array}{cc}
\left(A_{0}+\sum_{i=1}^{m} \lambda_{i} A_{i}\right) & \left(b_{0}+\sum_{i=1}^{m} \lambda_{i} b_{i}\right) / 2 \\
\left(b_{0}+\sum_{i=1}^{m} \lambda_{i} b_{i}\right)^{T} / 2 & \left(c_{0}+\sum_{i=1}^{m} \lambda_{i} c_{i}\right)-\gamma
\end{array}\right]
$$

We further constrain $\lambda$ to be non-negative, that is $\lambda_{i} \geq$ $0, i=1, \ldots, m$. Once the problem is in this form, we can apply modern semidefinite programming codes to solve this optimization problem and provide a bound on the optimal value. Importantly, these codes are able to exploit the sparse structure of the $A_{i}$ matrices to significantly reduce the computational effort required to solve these problems.

1) Choosing Search Directions: Given the ability to bound the extent of the feasible region in a given search direction, we can now consider the question of how those search directions should be chosen. A straightforward but effective technique that works quite well in the general case 
is to simply choose a set of uniformly spaced search directions parallel to the plane of projection. With this approach, the accuracy of the resulting convex approximation is simply a function of the number of search directions employed.

In the special case where all of the measurement constraints are convex we have proposed [9] a more sophisticated approach to choosing search directions which allows us to make precise statements about the performance of the projection approximation scheme.

\section{LOCALIZATION EXPERIMENTS}

The simulation experiments described in this section were designed to characterize the performance of the localization scheme under a variety of conditions. For each trial, a set of 10 nodes were randomly distributed on a plane. The $x$ and $y$ coordinates of these locations were restricted to the unit interval. A randomly chosen subset of the available range and bearing measurements relating the nodes were considered. These measurements were then corrupted with uniformly distributed, bounded random errors. The range measurements were corrupted with random errors in the range \pm 0.1 while the bearing measurements were corrupted with errors in the range $\pm 0.25^{\circ}$.

The magnitude of the angular errors was chosen by considering a typical camera with a 60 degree field of view and a horizontal resolution of 640 pixels. In this context a localization error of \pm 2 pixels on each bearing measurements would translate to an error of approximately 0.2 degrees. Note that this means that the relative bearing measurements would have errors in the range \pm 0.4 degrees. Importantly, we do not assume that the robots have access to an absolute bearing sensor like a compass, so the robots do not, initially, have any idea about their relative orientation.

The localization procedure described previously was used to construct convex approximations for the position of each of the nodes with respect to the first node which was fixed as the origin $(0,0)$. To fix orientation and scale, the second point in the set was also held fixed. The convex approximations for the remaining 8 node locations were constructed by considering 8 evenly spaced search directions in the plane.

For purposes of comparison, the localization procedure was run under four different conditions. The first variant only made use of the convex range constraints given in Equation 6 this experimental condition is analogous to the localization scheme described in [15]. The second condition made use of both the convex and non convex range constraints. The third condition only considered the relative bearing constraints while the last condition considered all available range and bearing constraints.

The results obtained on a typical trial are shown in Figure 7. Figure 6 shows the ground truth configuration used for this trial. Here the crosses denote the randomly chosen node positions and the edges indicate the available range and bearing measurements. A grand total of 51 range and bearing measurements were used in this instance.

In order to compare the results of these four conditions quantitatively, we computed the areas of the polyhedral

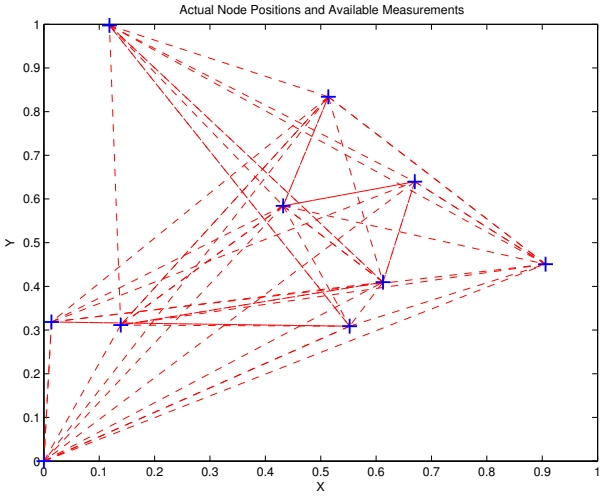

Fig. 6. For each trial a set of 10 node locations was chosen at random, these are denoted by the blue crosses. The edges between the nodes indicate available range and/or bearing measurements.

approximations returned by the procedure. These areas provide an indication of how effective the constraints are at narrowing down the set of feasible configurations. Larger areas correspond to greater uncertainty. The results of this analysis are summarized in Table I.

These results shows that under these experimental conditions, the relative bearing constraints are much more powerful than the range constraints. The convex approximations constructed with these constraints are two orders of magnitude smaller than those constructed using the range constraints. When the range constraints are added to the bearing constraints the average area is approximately halved.

In these experiments the results obtained with the range only localization scheme reflect the fundamental flip ambiguity associated with such measurements. That is, any configuration that satisfies the range constraints can be reflected in the plane to obtain another configuration that also satisfies the constraints. The convex bounding region that this method constructs must reflect that. In order to overcome this, one would need to presuppose the existence of known anchor points or some other means of resolving the ambiguity. In this sense range and bearing measurements can serve as complementary sources of information since the bearing measurements can resolve the flip ambiguity associated with the range measurements while the range measurements resolve the scale ambiguity associated with the bearing measurements.

In this round of simulation experiments the system did not attempt to estimate the relative orientations of the robots. It is possible to construct bounded uncertainty estimates for these quantities when bearing measurements are available as described in [26].

\section{DisCuSSION AND CONCLUSIONS}

This paper describes a novel approach to multi-robot localization grounded in robust estimation. The scheme employs an unknown-but-bounded error model for sensor measurements and leverages recent advances in convex optimization theory - specifically computational improvements 
a.
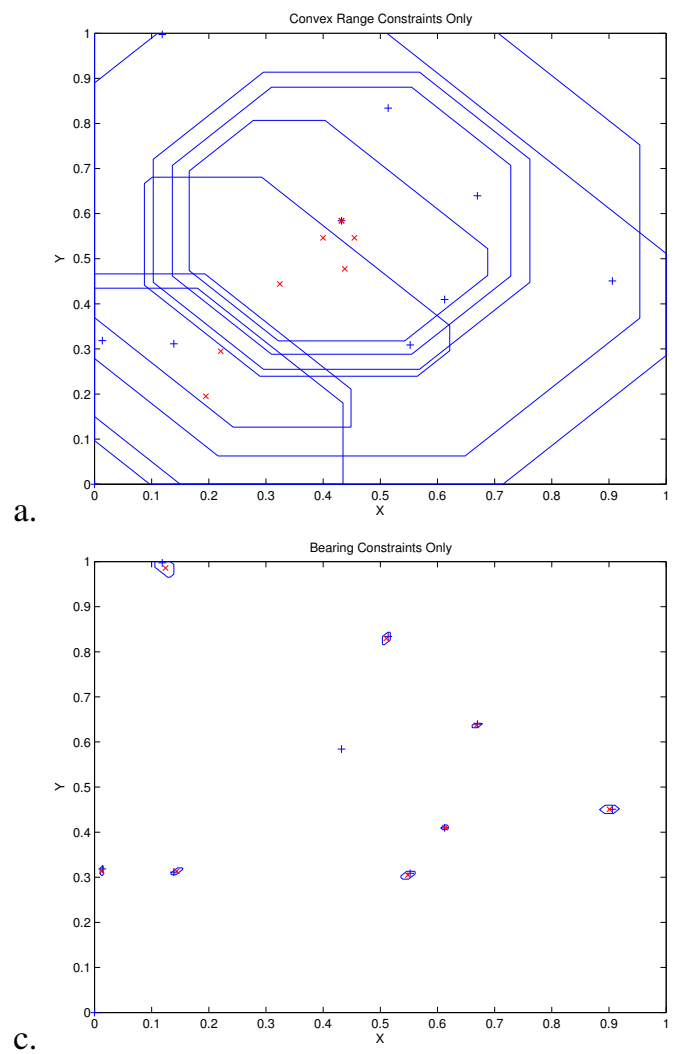

b.
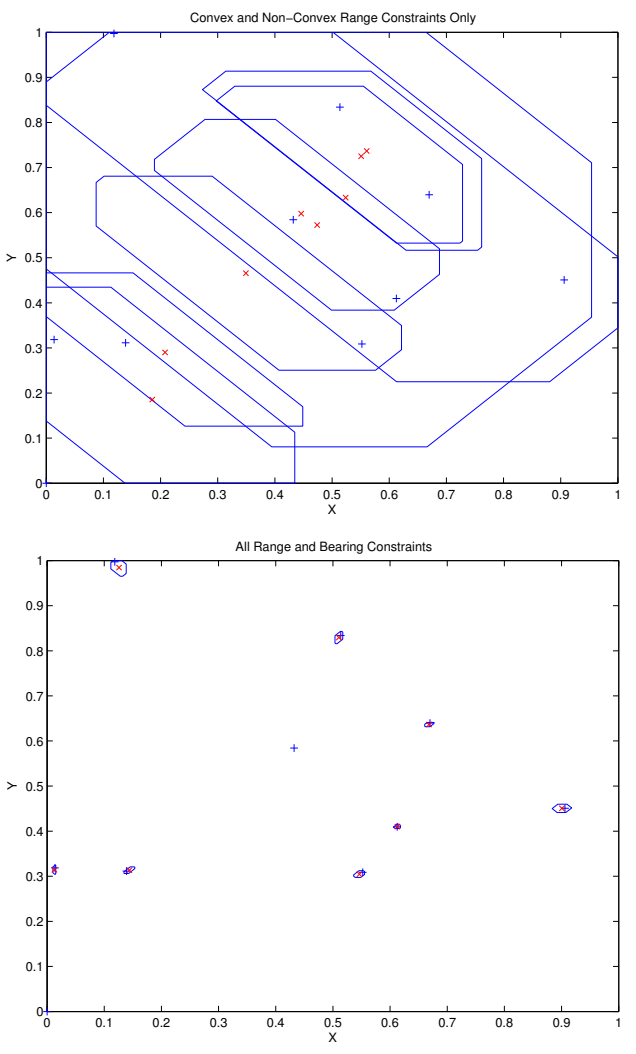

Fig. 7. The localization procedure was run under four conditions. a) Using only the convex range constraints b) Using both convex and non-convex range constraints. c) Using only the relative bearing constraints d) Using all available range and bearing constraints. The blue crosses in these figures denote the actual node locations while the red xs denote the centroids of the bounding polygons.

\begin{tabular}{l|c|c|c} 
Conditions & Mean Area & Median Area & Max. Area \\
\hline Convex Range Only & 0.4610 & 0.4433 & 0.9956 \\
Convex and NonConvex Range & 0.3540 & 0.3175 & 0.9805 \\
Relative Bearing Only & 0.0020 & 0.0008 & 0.0254 \\
All Constraints & 0.0011 & 0.0005 & 0.0108
\end{tabular}

TABLE I

COMPARISON OF BOUNDING AREAS $\mathcal{P}^{+}$OBTAINED UNDER VARIOUS EXPERIMENTAL CONDITIONS

in semidefinite programming techniques, duality, and Lagrangian relaxations - to provide a localization framework suitable for robotic systems.

Through convex approximations, our framework is able to integrate any mixture of range and/or bearing measurements from the robot formation into a single estimator which provides estimates for the uncertainty in node positions that are simultaneously conditioned on all available sensor measurements. Since the method combines measurements through set intersection rather than Bayes rules, it avoids many of the issues associated with accounting for interdependencies between multiple estimates. Furthermore, the resulting uncertainty regions are guaranteed to contain the true robot positions. Point estimates for the robots relative positions can be obtained by considering the centroids or Euclidean centers of various projections of the feasible set.

The ability to handle both convex and non-convex measurement constraints is an important advance. With this capability, we are able to make use of the relative bearing constraint which takes the form of a nonconvex quadratic inequality. This constraint is particularly useful since we can typically measure relative bearings quite accurately with imaging devices, often to within a small fraction of a degree. Absolute bearing measurements, such as those obtained from a compass are often off by a few degrees. Our localization scheme is able to generate accurate estimates for the configuration of a team of robots based on relative range and bearing constraints without any prior estimates for the robots orientation. This is potentially a significant advance over EKF schemes which must account for uncertainties in robot orientation through linearized approximations.

These advantages come at a cost however, and this is the computational complexity of the associated semidefinite programs. In theory, SDPs have an iteration complexity of $O\left(m^{\frac{5}{2}} n^{2}\right)$, where $n$ corresponds to the number of nodes and $m$ the number of constraints - or in our case sensor 
measurements [23]. However, it is well known that this bound is conservative and based upon empirical results in [15], we would expect the results to be closer to $O\left(n^{3}\right)$ in practice. This makes the approach suitable for real-time operations for reasonably large formation sizes (i.e., 10s of robots).

While the computational complexity of our approach significantly lags that of the EKF, it fares better with respect to storage complexity. The EKF requires $O\left(n^{2}\right)$ storage for representing the state covariance matrix. Assuming $k$ linear inqualities are used to model each uncertainty region, we require $O(k n)$ storage. However, our previous work in [9] showed that the quality of the approximation of the projection was independent of the number of nodes or sensor measurements. As a result, $O(n)$ storage complexity should be expected.

While the proposed technique has been described in the context of static nodes the method can be extended to dynamic nodes by convolving the uncertainty regions obtained at one instant with the uncertainties associated with the motion model to derive additional constraints on the node locations during subsequent timestep. We are also investigating the problem of outlier rejection since incorrect measurements with artificially small error bounds can result in inconsistent constraints and an empty feasible set. Here we may be able to employ techniques from robust estimation like validation gates which test a proposed measurement against an existing feasible set before acceptance. Another approach would be to choose random subsets of the available measurements to define the feasible set and identify outliers [27].

\section{ACKNOWLEDGMENTS}

The authors gratefully acknowledge the support of an ARO MURI DAAD-19-02-1-0383 “Adaptive Coordinated Control of Intelligent Multi-Agent Teams (ACCLIMATE)".

\section{REFERENCES}

[1] D. Fox, W. Burgard, H. Kruppa, and S. Thrun, "A probablistic approach to collaborative multi-robot localization," Autonomous Robots: Special Issue on Heterogeneous Multi-Robot Systems, vol. 8, no. 3, pp. 325-344, 2000.

[2] S. Majumder, S. Scheding, and H. Durrant-Whyte, "Multi-sensor data fusion for underwater navigation," Robotics and Autonomous Systems, vol. 35, no. 1, pp. 97-108, 2001.

[3] S. Thrun, "A probabilistic online mapping algorithm for teams of mobile robots," International Journal of Robotics Research, vol. 20, no. 5, pp. 335-363, 2001.

[4] D. Kurth, G. Kantor, and S. Singh, "Experimental results in rangeonly localization with radio," in IEEE/RSJ International Conf. on Intelligent Robots and Systems, Las Vegas, NV, Oct 2003.

[5] P. Newman and J. Leonard, "Pure range-only subsea slam," in IEEE International Conference on Robotics and Automation, Taiwan, China, Sep 2003.

[6] S. Roumeliotis and G. Bekey, "Collective localization: a distributed kalman filter approach," IEEE Transactions on Robotics and Autonomation, vol. 18, no. 5, pp. 781-795, 2002.

[7] A. Howard, M. Mataric, and G. Sukhatme, "Putting the 'i' in team: an ego-centric approach to cooperative localization," in Proceeding of the IEEE Int. Conference on Robotics and Automation, Taipei, Taiwan, May 2003.

[8] A.I. Mourikis and S.I. Roumeliotis, "Performance analysis of multirobot cooperative localization," IEEE Transactions on Robotics, vol. 22, no. 4, pp. 666-681, 2006.
[9] J. Spletzer and C.J. Taylor, "A bounded uncertainty approach to multirobot localization," in Proceedings of the Conference on Intelligent Robots and Systems (IROS 2003), 2003.

[10] S. Marsland T. Duckett and J. Shapiro, "Simultaneous localization and mapping - a new algorithm for a compass-equipped mobile robot," in Proceedings of IJCAI-2001 Workshop on Reasoning with Uncertainty in Robotics, Seattle, WA, Aug 2001.

[11] A. Georgiev and P. K. Allen, "Localization methods for a mobile robot in urban environments," IEEE Transactions on Robotics, vol. 20, no. 5, pp. 851-864, 2004.

[12] A.I. Mourikis Y.S. Hidaka and S.I. Roumeliotis, "Optimal formations for cooperative localization of mobile robots," in Proceeding of the IEEE Int. Conference on Robotics and Automation, Barcelona, Spain, April 2005, pp. 4137-4142.

[13] R. Kurazume and S. Hirose, "Study on cooperative positioning system," in Proc. IEEE Int. Conf. Robot. Automat., Leuven, Belgium, May 1998, pp. 2896-2903.

[14] Ioannis M. Rekleitis, Gregory Dudek, and Evangelos E. Milios, "Multi-robot collaboration for robust exploration," Annals of Mathematics and Artificial Intelligence, vol. 31, no. 1-4, pp. 7-40, 2001.

[15] L. Doherty, K. Pister, and L. El Ghaoui, "Convex position estimation in wireless sensor networks," in Proceedings of IEEE Infocom, April 2001.

[16] D. Moore, J. Leonard, D. Rus, and S. Teller, "Robust distributed network localization with noisy range measurements," in Proceedings of the Second International Conference on Embedded and Networked Sensor Systems, SenSys 04, November 2004, pp. 39-50.

[17] M. Paskin S. Funiak, C. Guestrin and R. Sukthankar., "Distributed localization of networked cameras," in ISPN, 2006.

[18] Pratik Biswas, Tzu-Chen Lian, Ta-Chung Wang, and Yinyu Ye, "Semidefinite programming based algorithms for sensor network localization," ACM Trans. Sen. Netw., vol. 2, no. 2, pp. 188-220, 2006.

[19] Babak Shirmohammadi Camillo J. Taylor, "Self localizing smart camera networks and their application to $3 \mathrm{~d}$ modeling," in ACM SenSys/First Workshop on Distributed Smart Cameras, October 2006.

[20] Hamid Aghajan Pratik Biswas and Yingu Ye, "Semidefinite programming algorithms for sensor network localization using angle information," in Asilomar Conference on Signals, Systems and Computers, October 2005.

[21] Mauro Di Marco, Andrea Garulli, Antonio Giannitrapani, and Antonio Vicino, "Simultaneous localization and map building for a team of cooperating robots: A set membership approach," IEEE Transactions on Robotics and Automation, vol. 19, no. 2, pp. 238-249, April 2003.

[22] K. Briechle and U. D. Hanebeck, "Localization of a mobile robot using relative bearing measurements," IEEE Transactions on Robotics and Automation, vol. 20, no. 1, pp. 36-44, February 2004.

[23] L. Vandenberghe and S. Boyd, "Semidefinite programming," SIAM Review, 1996.

[24] S. Boyd and L. Vandenberghe, Communications, Computation, Control and Signal Processsing: a Tribute to Thomas Kailath, chapter Semidefinite programming relaxations of non-convex problems in control and combinatorial analysis, Kluwer, 1997.

[25] S. Boyd and L. Vandenberghe, Convex Optimization, Cambridge University Press, 2004.

[26] A. Das, J. Spletzer, V. Kumar, and C. J. Taylor, "A distributed multirobot system for cooperative manipulation," in Multi-Robot Systems: From Swarms to Intelligent Automata, 2002.

[27] M. Fischler and R. Bolles, "Random sample consensus: A paradigm for model fitting with applications to image analysis and automated cartography," in Communications of the ACM, 1981. 